Preprints are preliminary reports that have not undergone peer review. They should not be considered conclusive, used to inform clinical practice, or referenced by the media as validated information.

\title{
SAA4 is a Diagnostic Marker to Enhance Detection Forrheumatoid Arthritis Combined with Anti-CCP
}

\section{Jiahui Li}

Department of Clinical Epidemiology and Evidence-based Medicine, the First Affiliated Hospital, China Medical University,Shenyang

\section{Haina Liu}

Department of Rheumatology, the First Affiliated Hospital, China Medical University, Shenyang

\section{Bingbing Dai}

Department of Rheumatology, Dalian Municipal Central Hospital Affiliated of Dalian Medical University, Dalian

\section{Zhijun Fan}

Department of Clinical Epidemiology and Evidence-based Medicine, the First Affiliated Hospital, China Medical University, Shenyang

\section{Qiao Wang}

Department of Clinical Epidemiology and Evidence-based Medicine, the First Affiliated Hospital, China Medical University,Shenyang

\section{Huiyuan Liu}

Department of Clinical Epidemiology and Evidence-based Medicine, the First Affiliated Hospital, China Medical University, Shenyang

\section{Tingting Wei}

Department of Clinical Epidemiology and Evidence-based Medicine, the First Affiliated Hospital, China Medical University,Shenyang

\section{fangran Xin}

Department of Clinical Epidemiology and Evidence-based Medicine, the First Affiliated Hospital, China Medical University,Shenyang

\section{Bingqing Bai}

Department of Clinical Epidemiology and Evidence-based Medicine, the First Affiliated Hospital, China Medical University, Shenyang

\section{Lingyu Fu ( $\nabla$ fulingyucmu@sina.com )}

Department of Medical Record Management Center, the First Affiliated Hospital, China Medical University, Shenyang

\section{Research Article}

Keywords: rheumatoid factor, Serum amyloid A4 (SAA4), anti-CCP, diagnostic biomarker, diagnostic model

Posted Date: January 6th, 2022

DOI: https://doi.org/10.21203/rs.3.rs-1221628/v1

License: (c) (i) This work is licensed under a Creative Commons Attribution 4.0 International License. Read Full License 


\section{Abstract}

Objective Serum amyloid A4 (SAA4) is an apolipoprotein that is associated with high-density lipoprotein (HDL) in plasma. In this present investigation, we appraised the potential of SAA4 as a novel diagnostic biomarker for rheumatoid arthritis (RA)

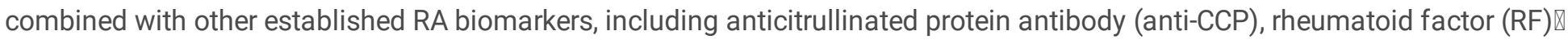
and C-reactive protein (CRP). Based on the correlative measures of the biomarkers, we developed a diagnostic model of RA by integrating serum levels of SAA4 with these clinical parameters.

Methods A number of 316 patients were recruited in the current research. The serum levels of SAA4 were assessed by quantitative ELISA. The specificity and sensitivity of biomarkers were evaluated by using a receiver-operator curve (ROC) analysis to determine their diagnostic efficiency. Univariate and multivariate logistic regression analyses were used to screen and construct the diagnostic models for RA , consisting of diagnostic biomarkers and clinical data. A diagnostic nomogram was then generated based on logistic regression analysis results.

Results The serum levels of SAA4 were considerably greatest in RA patients in comparison to other control subjects $(P<0.001)$. Compared with anti-CCP, RF and CRP respectively, SAA4 had the highest specificity (88.60\%) for diagnosing RA. The combination of SAA4 with anti-CCP could have the highest diagnostic accuracy when paired together, with highest sensitivity $(91.14 \%)$ in parallel and highest specificity(98.10) in series. We successfully developed two diagnostic models: the combined model of SAA4 and anti-CCP (model A), and the combined model of SAA4, CRP, anti-CCP, RF and history of diabetes (model B). Both models showed a great area under the curve of ROC for either the training cohort or the validation cohort. The data indicated that the novel RA diagnostic models possessed an advantageous discrimination capacity and application potential.

Conclusion Serum SAA4 has utility as a biomarker for RA's diagnosis and can enhance the detection of RA when combined with anti-CCP.

\section{Introduction}

RA is a chronic progressive disorder that mainly influences the joints. It is characterized by multiple clinical manifestations including inflammation, painful joints, persistent synovitis, degradation of the bone and cartilage and stiffness following prolonged periods of inactivity ${ }^{[1,2]}$. RA affects around $1 \%$ of the people worldwide and is more pervasive in women than men at a ratio of around $3: 1^{[3-5]}$. The treatment of RA remains challenging in the clinic as it generally requires lifelong treatment after the disease has fully developed. Patient management strategies using antirheumatic therapy are more effective when prescribed at the early stages of the disease, offering a therapeutic window of opportunity ${ }^{[6]}$. To fully exploit the benefits of a therapeutic window, the early diagnosis of RA is critical.

Currently, RA diagnostic methods is on the basis of the criteria published in 2010 by the European League Against Rheumatism (EULAR) and the American College of Rheumatology (ACR), ${ }^{[7]}$ which include the following diagnostic items: symptoms duration, serological and acute-phase response biomarkers abnormal levels such as rheumatoid factor (RF) and anticitrullinated peptide antibodies (anti-CCP) and site of the affected joints. Unfortunately, the diagnosis of RA is often not straightforward at the early phases of disease development, when some patients' clinical symptoms are not obvious enough to be identified. Abnormal biomarkers abnormal levels have become an important indicator for the early diagnosis of RA, and the criteria published guiding rules for the utilization of serum biomarkers, which included RF and anti-CCP ${ }^{[8,9]}$, no doubt also highlighting the biomarkers' utility in RA. However, the accuracy of current biomarkers are sub-optimal for early and accurate diagnosis of RA, highlighting the need for novel biomarkers with improved sensitivity and accuracy ${ }^{[10]}$. There is a critical unmet need for the development of improved biomarkers that can improve the early identification of RA and better inform clinical decision-making.

SAA4 is a part of the serum amyloid A (SAA) family that of acute phase proteins that are typically increased in response to a variety of inflammatory conditions ${ }^{[11]}$. Using proteomics analysis, Seok ${ }^{[12]}$ et al found that SAA4 levels were substantially raised in the serum of RA patients, as did Mun and colleagues ${ }^{[13]}$, who also showed that SAA4 has a high potential as a 
diagnostic biomarker for RA. Despite the evidence presented in these studies, additional investigations are essential to demonstrate the clinical value and practicality of using SAA4 as a biomarker for RA.

In this research, our main aim is to assess SAA4 as a diagnostic biomarker for RA. Additionally, this investigation will also aim to demonstrate the increased value of serum SAA4 in improving RA diagnosis when combined with established clinical parameters and serum biomarkers in RA. Our study provides important further understanding to support the clinical use of SAA4 in RA patients and proposes a novel model for RA diagnosis.

\section{Material And Methods}

\section{Patient selection}

The clinical data were collected and analyzed from outpatient or inpatient cases at the First Affiliated Hospital of China Medical University and Dalian Municipal Central Hospital Affiliated of Dalian Medical University from April 2017 to September 2019. Before recruitment, the sample size for the study was calculated using previous pre-test data according to the following diagnostic test equation ${ }^{[14]}$;

$$
N=\frac{z_{\alpha / 2}^{2} \pi(1-\pi)}{d^{2}}
$$

\section{In this formula, $z_{\alpha / 2}^{2}$ represents the $\alpha / 2$ quantile of the standard normal}

distribution, which is inserted by was 1.96 , and $d^{2}$ represents the allowable error which was 0.1 . $\Pi$ as determined by the value of sensitivity (or specificity) from the previous pre-test data which gave a value of 0.75 (the sensitivity and specificity were $75 \%$ and $60 \%$ in the pre-test). The required sample size was calculated to be at least 92 patients. We recruited RA patients into the RA group, patients with non-RA autoimmune disease into the non-RA disease control group, and healthy cases into the healthy control group.

For the recruitment of RA patients in the research, the following inclusion criteria were established; (1) Patients who met the diagnostic parameters for the RA proposed by the ACR (1987/2010); 2) RA patients diagnosed for the first time; (3) Patients over 18 years old. Two control groups were selected, one was the non-RA control group, the other was the healthy control group. The non-RA control group consisted of patients with dignosis that may easily confused with RA, such as systemic lupus erythematosus (SLE), primary Sjogren syndrome(pSS) and ankylosing spondylitis (AS). For the recruitment of non-RA disease patients in the research, the following inclusion criteria were established; (1) Patients who met the diagnostic parameters proposed by the ACR and the EULAR ${ }^{[15-17]}$ for SLE, pSS and AS respectively; (2) Patients who had the first diagnosis of non-RA diseases; (3) Patients over 18 years old. Healthy controls were recruited based on the professional assessment of clinicians. Patients were excluded if they lacked routine laboratory assessment data, including antibodies or diagnostic biomarkers associated with RA. Based on the estimation of sample size and the particular inclusion and exclusion criteria, a number of 316 patients were recruited in the investigation at last. This study was approved by the medical research ethics committee of the first affiliated hospital of China medical university (AF-SOP-07-1.0-01).

\section{Clinical information}

For each patient, data were collected on their demographic baseline characteristics and laboratory results diagnostic indicators. The demographic baseline characteristics included gender, age, smoking and drinking habits, and any history of hypertension and diabetes. The laboratory data recorded the level of biomarkers in the sera of patients obtained as part of routine diagnostic evaluation, including CRP, anti-CCP, and RF antibodies. All laboratory data were measured at the first time of presentation. Patients were stratified based on their smoking habits defined as either long-term regular smoker ( $\geq 1$ one 
cigarette per day) or infrequent smokers, defined as those who have never smoked or had given up smoking for $>1$ year. Patients were also stratified based on their alcohol drinking habits, with long-term drinker defined as those who consume $\geq 1$ measure of liquor or bottle of beer per day for one year, and infrequent drinkers defined as those who had never consumed alcohol or had given up drinking for more than one year.

\section{Measurement of biomarkers}

A $5 \mathrm{ml}$ sample of fasting venous was collected from each participants. Blood specimens were permitted to clot at $37^{\circ} \mathrm{C}$ before being centrifuged at $3000 \mathrm{rpm}$ for 10 minutes. The sera were separated and immediately frozen at $-80^{\circ} \mathrm{C}$ immediately until further examination. The serum concentrations of SAA4 were determined through ELISA using assay reagents supplied by Qiantu (Shanghai, China). The levels of the serum biomarkers (RF, anti-CCP and CRP) were determined using ELISA kits obtained from the same supplier (Qiantu). All assays were performed according to the instructions of the manufacturer. Different threshold levels of the serum biomarkers were used to define positive tests. Specifically, $\geq 20 \mathrm{IU} / \mathrm{mL}$ for $R F, \geq 17$ $\mathrm{IU} / \mathrm{mL}$ for anti-CCP and $\geq 8 \mathrm{mg} / \mathrm{L}$ for CRP.

\section{Statistical analysis}

The continuous categorical variables were expressed as mean \pm standard deviation when they fit the normal distribution. If the data has an asymmetrical distribution, the continuous categorical variables were expressed as medians (interquartile range), and the categorical variables were expressed as number and proportion as appropriate. For inter-group comparisons of clinical characteristics, Wilcoxon's rank-sum test and chi-squared test were applied for continuous variables and count data, respectively. The diagnostic power for biomarkers in recognition RA was estimated using receiver operating characteristics (ROC) curve and area under the curve (AUC).

Diagnostic models were generated by dividing the study group into training cohort and validation cohort firstly. The training cohort was comprised of $70 \%$ of the total study cohort (224 patients) and was used to construct the diagnostic models of RA. The validation cohort was comprised of the remaining $30 \%$ of the total study cohort (92 patients) and was used to validate the diagnostic model.

The diagnostic models were constructed through first doing univariate logistic regression analysis on the training cohort data in order to identify the relationships between clinical biomarkers and criteria as an independent variable and RA as a dependent variable. To minimize the potential influence of confounding factors, the relationships between determined dependent and independent variables may be undetectable in univariate analysis. The variables with P-values $<0.100$ were included into the analysis of multivariate logistic regression.

We developed the individualized nomogram diagnosis prediction model of RA based on the results of regression analysis. Besides, we evaluated the diagnosis models in terms of discrimination, which is most often assessed by calculating AUC of the ROC curve ${ }^{[18]}$. The models were then tested in the validation cohort utilizing the identical regression equations created in the training cohort.

All statistical analyses were carried out utilizing SPSS (ver23.0, USA), R software (ver3.4.0, USA) and SigmaPlot (ver13.0, USA). The considered two-sided $P$-values $<0.05$ were statistically significant.

\section{Results}

\section{Characteristics of the study population}

A total of 316 patients were enrolled in our study, including 158 RA patients in the RA group, 45 healthy controls, and 113 nonRA patients (SLE, $n=49 ; p S S, n=14 ; A S, n=50$ ) in the disease controls. The research population's clinical, immunological and 
demographic characteristics are summarized in Table 1. The entire cohort consisted of 256 females and 60 males. The mean age of RA patients was $52.35 \pm 12.93$ years, $51.21 \pm 14.41$ for non-RA disease patients and $55.22 \pm 7.48$ for healthy controls. The age and gender of RA patients and all the controls were matched. There were no considerable differences in age, gender, smoking history and history of alcohol consumption observed between the RA group, the non-RA controls and the healthy controls. However, the history of diabetes and hypertension were remarkably distinct between the groups.

\section{Serum levels of SAA4, RF, anti-CCP and CRP}

The serum level of the biomarkers was compared between the RA group and the two controls. As shown in Table 1, the median (IQR) serum concentration of SAA4 in the RA groupwas $62.53 \mu \mathrm{g} / \mathrm{ml}$, which was substantially greater than the $44.03 \mu \mathrm{g} / \mathrm{ml}$ observed in the disease controls and $39.15 \mu \mathrm{g} / \mathrm{ml}$ reported in the healthy controls. This difference of serum concentration between the groups was statistically significant $(P<0.001)$. The levels of CRP, anti-CPP and RF were also notably higher in RA patients compared to the other patient groups $(P<0.001)$.

\section{Potential diagnostic values of SAA4}

The sensitivity, specificity, Youden index, positive likelihood ratio, negative likelihood ratio, positive predictive value and negative predictive value of SAA4 were all tested to determine its diagnostic utility as a biomarker for RA. Simultaneously, we measured the corresponding statistical indicators being listed above for RF, anti-CCP and CRP, and then estimated the AUC of the ROC to compare different biomarkers' discriminative ability.

When restricted to the RA patient group, as shown in Table 2, SAA4 demostrated the highest specificity (88.60\%) compared to the other biomarkers. Compared to RF, anti-CCP, and CRP, SAA4 is higher specific (88.60\%) but less sensitive (67.72\%). Besides, SAA4 also demonstrated favorable positive likelihood ratio (5.94) and the positive predictive value (0.86). In addition, the YI of SAA4 was 0.56 indicating good accuracy, authenticity and reliability; and it is of outstanding value in the diagnosis of RA. When comparing the RA group to the disease controls, the healthy controls or the two controls combined, as shown in Figure 1,SAA4 had significantly greater AUC than other biomarkers (all $p<0.005$ ), which indicates its outstanding diagnostic accuracy. According to the ROC analysis, the optimum cut-off level of SAA4 was $56.14 \mu \mathrm{g} / \mathrm{ml}$ with a sensitivity of $67.72 \%$ and a specificity of $88.60 \%$.

The incremental benefit of combining SAA4 with the other routine biomarkers was assessed using a variety of biomarkers combinations. In terms of overall diagnostic accuracy, the combination of four biomarkers, namely SAA4, RF, anti-CCP and CRP, had the highest sensitivity (97.47\%) and specificity (99.37\%) When considering the combination of two biomarkers, the sensitivity and specificity also significantly increased in comparison to when any biomarker is considered alone (shown in Table 3). When SAA4 was combined with anti-CCP, both the sensitivity $(91.14 \%)$ when used in parallel and the specificity $(98.10 \%)$ when used in series were highest among all the two biomarkers' combinations.

\section{Diagnostic model}

The baseline characteristics of the validation and training cohorts are summarized in Table 4. The training cohort consisted of 224 cases (112 RA patients and 112 controls) and the validation cohort consisted of 92 cases (46 RA patients and 46 controls). The results of univariate and multivariate logistic regression analyses were applied to contrast the RA diagnostic models and were used to build the final model, as shown in Table 5. The final parameters selected by multivariate logistic regression anlysis for the diagnostic model were SAA4, CRP, anti-CCP , RF and history of diabetes.

Based on the multivariate logistic regression analysis results, we constructed two types of diagnostic models in 224 training samples: a combined model of SAA4 and anti-CCP (model A), and a combined model of SAA4, CRP, anti-CCP, RF and history of diabetes (model B). Both two models showed favorable performance for the diagnosis of RA. The curves of ROC for the 
training and validation cohorts are presented in Fig 2. In the training cohort, the AUCs of model A and model B were $0.90[95 \% \mathrm{Cl}: 0.86-0.94]$ and $0.93[95 \% \mathrm{Cl}: 0.91-0.96]$ respectively. This similar results in the validation cohort diagnostic models suggested that the two models were stable. It is worth noting that SAA4, when paired up with anti-CCP, showed great diagnosis power, which was similar to the combined model of SAA4 and CRP, anti-CCP, RF and clinical parameters.

Furthermore, individualized nomograms of RA diagnostic model were developed with the intention of using the diagnostic models in clinical settings (Fig 3). Due to the wide range of OR $95 \% \mathrm{Cl}$ of diabetes, the final diagnostic model was constructed after adjusting for the history of diabetes. This can be demonstrated by the example of a 72-year-old female who tested positive for all four biomarkers, including SAA4, RF, anti-CCP and CRP. In terms of the cumulative score of the different prediction indicators, the corresponding predicted risk of RA in model A was 96.5\%, while in model B was 98.6\% (Fig 4). Both models showed high-risk of RA based on the predicted probability, and the results were similar. Between these two models, model A seems to be a more convenient tool to diagnose RA because it has less index.

\section{Discussion}

In the current investigation, we demonstrated the utility of SAA4 as a potential diagnostic biomarker for RA and compared the accuracy of diagnostic models integrating SAA4 with the established clinical parameters. SAA4 shows great detection power for RA when combined with anti-CCP. To the best of our knowledge, this is the first report to evaluate the diagnostic value of the SAA4 as part of a novel diagnostic model for RA.

The identification and development of novel diagnostic biomarkers remain a major area of interest in many diseases, including RA. SAA4 is a part of the serum amyloid SAA family of proteins that are seen in the acute-phase. Currently, four SAA subtypes associated with cholesterol homeostasis have been identified ${ }^{[19-21]}$. SAA1 and SAA2 are acute phase apolipoproteins that increase by $>100$-fold during acute inflammation and are correlated with the proteins involved in the regulation of angiogenic factors, such as matrix metalloproteinase $1(\mathrm{MMP} 1)^{[22]}$. SAA3, on the other hand, although is represented in mice only and has no human homolog, has long been regarded as a pseudogene. In contrast, the role of SAA4 in human diseases is limited, as

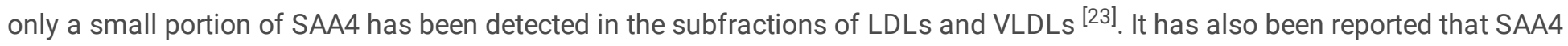
expression has been observed in ovarian tumors ${ }^{[24]}$ and may serve as a serologic and histologic biomarker for atheromatous lesions ${ }^{[25]}$. Seok et al. ${ }^{[12]}$ were the first to report SAA4 as a potential biomarker in RA. They showed that SAA4 was differentially expressed in the serum of RA patients using an LC-MS/MS proteomics approach. These findings were further validated by Mun et al. ${ }^{[13]}$, reporting that the upregulation of SAA4 in patients with RA when compared to healthy controls. Taken together, these results present a strong rationale for the potential use of SAA4 as a novel diagnostic biomarker for RA.

Our research focused on assessing the diagnostic value and clinical applicability of SAA4 in RA. In general, SAA4 showed a good diagnostic performance. The result showed that the expression of SAA4 was significantly increased in serum of RA patients compared to all the controls. This finding indicates that the serum level of SAA4 might be an indicator of RA. Compared to RF, anti-CCP, and CRP, SAA4 is more specific but less sensitive. SAA4 might be a reasonably specific biomarker of RA. The largest AUC in SAA4 compared to the other biomarkers suggested that SAA4 levels could more accurately differentiate future RA patients from those who did not develop the disease. This discriminatory capacity of SAA4 exceeded the values for the other biomarkers, indicating once again that serum SAA4 may be a novel and highly valuable diagnostic indicator for RA.

The role of anti-CCP, RF, and CRP in the diagnosis of RA is now well established. Some researchers reported discrepancies between their assessment results of the diagnostic value thus far ${ }^{[26-31]}$, and the sensitivity and specificity of anti-CCP were from $60 \%$ to $70 \%$ and $70 \%$ to $97 \%$, respectively, and corresponding values of RF were $60 \%-83 \%$ and $70 \%-90 \%$, respectively. Our results were consistent with previous studies on the performance of anti-CCP and RF and showed good diagnostic utility. However, the results of different studies varied greatly for CRP. One study reported that CRP had a sensitivity of $91.3 \%$ and a specificity of $37.8 \%{ }^{[28]}$, the other study reported that CRP had a sensitivity of $72.7 \%$ and a specificity of $95 \%{ }^{[31]}$. Our study showed that the sensitivities of CRP were $58.22 \%$ with corresponding specificities of $78.48 \%$. These differences might be explained by the heterogeneity of the participants in the studies. 
As we all known, the joint test can increase diagnostic power over single diagnostic test ${ }^{[22,33]}$. In our study, the joint test's results suggested that the highest sensitivity and specificity were obtained when SAA4 and anti-CCP were combined together as diagnostic candidate biomarkers among all the biomarkers pairwise combinations. The combination test of SAA4 and anti-CCP greatly enhance detection of RA than either of them measured independently. The use of SAA4 as a novel biomarker combined with anti-CCP may be a cost-effective way to improve RA patients' diagnosis, which reduce the financial burden of patients. Also, the high level of accuracy obtained when using the combined biomarkers may also have important health economics benefits, which may offset some limitations of routine biomarkers. The patient may achieve the best diagnostic effect at a lower cost. While combined analyses of various biomarkers and clinical data are more accurate and have the potential to provide greater patient benefit ${ }^{[30]}$.

The statistical modeling process aims to create a simplified description of the object of study in order to achieve a certain purpose $^{[34]}$. Diagnostic models are becoming increasingly being used in many areas of medicine as a method to improve the accuracy of clinical diagnosis. In this study, diagnostic models for RA were developed using real-world data in conjunction with established clinical biomarkers and SAA4. By comparing our models, it was found that when SAA4 or clinical parameters were combined, the diagnostic performance of SAA4 or clinical parameters alone was improved, and the combination enhanced the capability of RA to be diagnosed early. Our results suggested that SAA4 positivity was an independent diagnosis factor in RA patients. The studied diagnostic model combined several indicators (CRP, anti-CCP, RF and SAA4) and demostrated diagnostic capacity in both validation and training cohorts, indicating that the model was stable. Consistent with other studies ${ }^{[30,35]}$, our results highlighted the important advantages of combining multiple biomarkers over single diagnostic parameters and provided a novel method for combining them. Nomogram is a reliable tool for informing clinical decision-making. Our nomogram might be applied to outpatients as a supplementary tool for clinical evaluation and could assist patients in understanding their diagnostic decision-making processes.

Despite the positive findings in our study, there were still several limitations. While our sample size was sufficiently large and statistically robust, most of the patients recruited to the study came from northern China. Also, our findings require further validation in prospective studies in order to provide a more comprehensive and in-depth clinical evaluation of our prediction model.

\section{Conclusion}

our results suggested that serum SAA4 levels could be used as a diagnostic biomarker for RA with a discriminative ability comparable to that of routine biomarkers. The combination SAA4 with anti-CCP greatly enhanced detection for RA. Also, an individualized nomogram diagnostic prediction model was established that can be used to accurately predict RA. The earlier and more accurate diagnosis of RA may have a high potential to improve outcomes for patients by identifying people who need disease modifying therapy and hence to minimize the health and economic costs of RA.

\section{Abbreviations}

RA:rheumatoid arthritis;ACR:American College of Rheumatology; anti-CCP:anti-cyclic citrullinated peptide antibody; antiCarP:Antibodies against carbamylated proteins;APF :Antiperinuclear factor autoantibody;AS:Ankylosing spondylitis;AUC :Area under ROC;ELISA:Enzyme linked immunosorbent assay;EULAR :European League Against Rheumatism;IL-6 :Interleukin6;OR:Odds ratio;pSS:Primary Sjogren syndrome;SAA4 :Serum amyloid A4;SLE:Systemic lupus erythematosus;RF:rheumatoid factor; CRP:C-reactive protein; ESR:erythrocyte

\section{Declarations}

\section{Ethics approval and consent to participate}


The study protocol was approved by Institutional Medical Ethics Review Board of the First Affiliated Hospital of China Medical University (AF-SOP-07-1.0-01). Informed written consent was obtained from all study participants according to the principlesof the Declaration of Helsinki.

\section{Consent for publication}

All of the subjects provided written informed consent for publication.

\section{Availability of data and materials}

The datasets used and or analyzed during the current study are available

from the corresponding author on reasonable request.

\section{Competing interests}

The authors declare that they have no competing interests.

\section{Funding}

This work was supported by National Natural Science Foundation of China (Grant No. 82173604), The funders had no role in study design, data collection and analysis, decision to publish, or preparation of the manuscript.

\section{Authors'contributions}

$\mathrm{JH}$ conceived of the study and participated in its design and coordination. JH carried out the ELISA and performed the statistical analysis. BB, HN,BQ,FY and TT collected samples. HY, ZJ, and QW contributed to data acquisition, analysis, and critical review for intellectual content. JH and LY drafted the manuscript and revised the manuscript. All authors read, revised, and approved the final manuscript.

\section{Acknowledgements}

We thank all participating subjects who provided blood samples and clinical information necessary for this study.

\section{References}

1. SMOLEN J S, ALETAHA D, MCINNES I B. Rheumatoid arthritis [J]. Lancet (London, England), 2016, 388(10055): $2023-38$.

2. ALAMANOS Y, DROSOS A A. Epidemiology of adult rheumatoid arthritis [J]. Autoimmunity reviews, 2005, 4(3): 130-6.

3. GóMEZ R, CONDE J, SCOTECE M, et al. What's new in our understanding of the role of adipokines in rheumatic diseases? [J]. Nature reviews Rheumatology, 2011, 7(9): 528-36.

4. SCOTT D L, WOLFE F, HUIZINGA T W. Rheumatoid arthritis [J]. Lancet (London, England), 2010, 376(9746): 1094-108.

5. GABRIEL S E. The epidemiology of rheumatoid arthritis [J]. Rheumatic diseases clinics of North America, 2001, 27(2): 26981.

6. FINCKH A, ALPIZAR-RODRIGUEZ D, ROUX-LOMBARD P. Value of Biomarkers in the Prevention of Rheumatoid Arthritis [J]. Clinical pharmacology and therapeutics, 2017, 102(4): 585-7. 
7. ALETAHA D, NEOGI T, SILMAN A J, et al. 2010 Rheumatoid arthritis classification criteria: an American College of Rheumatology/European League Against Rheumatism collaborative initiative [J]. Arthritis and rheumatism, 2010, 62(9): 2569-81.

8. BERGLIN E, DAHLQVIST S R. Comparison of the 1987 ACR and 2010 ACR/EULAR classification criteria for rheumatoid arthritis in clinical practice: a prospective cohort study [J]. Scandinavian journal of rheumatology, 2013, 42(5): 362-8.

9. KOURILOVITCH M, GALARZA-MALDONADO C, ORTIZ-PRADO E. Diagnosis and classification of rheumatoid arthritis [J]. Journal of autoimmunity, 2014, 48-49(26-30.

10. BURR M L, VIATTE S, BUKHARI M, et al. Long-term stability of anti-cyclic citrullinated peptide antibody status in patients with early inflammatory polyarthritis [J]. Arthritis research \& therapy, 2012, 14(3): R109.

11. TARGOŃSKA-STĘPNIAK B, MAJDAN M. Response to: comment on "serum amyloid a as a marker of persistent inflammation and an indicator of cardiovascular and renal involvement in patients with rheumatoid arthritis" [J]. Mediators of inflammation, 2015, 2015(749565.

12. SEOK A, LEE H J, LEE S, et al. Identification and Validation of SAA4 as a Rheumatoid Arthritis Prescreening Marker by Liquid Chromatography Tandem-mass Spectrometry [J]. Molecules (Basel, Switzerland), 2017, 22(5):

13. MUN S, LEE J, LIM M K, et al. Development of a Novel Diagnostic Biomarker Set for Rheumatoid Arthritis Using a Proteomics Approach [J]. BioMed research international, 2018, 2018(7490723.

14. HAJIAN-TILAKI K. Sample size estimation in diagnostic test studies of biomedical informatics [J]. Journal of biomedical informatics, 2014, 48(193-204.

15. PETRI M, ORBAI A M, ALARCóN G S, et al. Derivation and validation of the Systemic Lupus International Collaborating Clinics classification criteria for systemic lupus erythematosus [J]. Arthritis and rheumatism, 2012, 64(8): $2677-86$.

16. SHIBOSKI C H, SHIBOSKI S C, SEROR R, et al. 2016 American College of Rheumatology/European League Against Rheumatism Classification Criteria for Primary Sjögren's Syndrome: A Consensus and Data-Driven Methodology Involving Three International Patient Cohorts [J]. Arthritis \& rheumatology (Hoboken, NJ), 2017, 69(1): 35-45.

17. VAN DER LINDEN S, VALKENBURG H A, CATS A. Evaluation of diagnostic criteria for ankylosing spondylitis. A proposal for modification of the New York criteria [J]. Arthritis and rheumatism, 1984, 27(4): 361-8.

18. HARRELL F E, JR., CALIFF R M, PRYOR D B, et al. Evaluating the yield of medical tests [J]. Jama, 1982, 247(18): 2543-6.

19. NAKARAI H, YAMASHITA A, NAGAYASU S, et al. Adipocyte-macrophage interaction may mediate LPS-induced low-grade inflammation: potential link with metabolic complications [J]. Innate immunity, 2012, 18(1): 164-70.

20. ROSSMANN C, WINDPASSINGER C, BRUNNER D, et al. Characterization of rat serum amyloid A4 (SAA4): a novel member of the SAA superfamily [J]. Biochemical and biophysical research communications, 2014, 450(4): 1643-9.

21. TARGOŃSKA-STĘPNIAK B, MAJDAN M. Serum amyloid A as a marker of persistent inflammation and an indicator of cardiovascular and renal involvement in patients with rheumatoid arthritis [J]. Mediators of inflammation, 2014, 2014(793628.

22. MULLAN R H, BRESNIHAN B, GOLDEN-MASON L, et al. Acute-phase serum amyloid A stimulation of angiogenesis, leukocyte recruitment, and matrix degradation in rheumatoid arthritis through an NF-kappaB-dependent signal transduction pathway [J]. Arthritis and rheumatism, 2006, 54(1): 105-14.

23. DE BEER M C, YUAN T, KINDY M S, et al. Characterization of constitutive human serum amyloid A protein (SAA4) as an apolipoprotein [J]. Journal of lipid research, 1995, 36(3): 526-34.

24. KRISTJANSDOTTIR B, PARTHEEN K, FUNG E T, et al. Ovarian cyst fluid is a rich proteome resource for detection of new tumor biomarkers [J]. Clinical proteomics, 2012, 9(1): 14.

25. FERNáNDEZ J A, DEGUCHI H, ELIAS D J, et al. Serum amyloid A4 is a procoagulant apolipoprotein that it is elevated in venous thrombosis patients [J]. Research and practice in thrombosis and haemostasis, 2020, 4(2): 217-23.

26. RIEDEMANN J P, MUñOZ S, KAVANAUGH A. The use of second generation anti-CCP antibody (anti-CCP2) testing in rheumatoid arthritis-a systematic review [J]. Clinical and experimental rheumatology, 2005, 23(5 Suppl 39): S69-76. 
27. SUN J, ZHANG Y, LIU L, et al. Diagnostic accuracy of combined tests of anti cyclic citrullinated peptide antibody and rheumatoid factor for rheumatoid arthritis: a meta-analysis [J]. Clinical and experimental rheumatology, 2014, 32(1): 1121.

28. SHOVMAN O, GILBURD B, ZANDMAN-GODDARD G, et al. The diagnostic utility of anti-cyclic citrullinated peptide antibodies, matrix metalloproteinase-3, rheumatoid factor, erythrocyte sedimentation rate, and C-reactive protein in patients with erosive and non-erosive rheumatoid arthritis [J]. Clinical \& developmental immunology, 2005, 12(3): 197-202.

29. KUROWSKA W, PRZYGODZKA M, JAKUBASZEK M, et al. Interleukin-15 as a Biomarker Candidate of Rheumatoid Arthritis Development [J]. Journal of clinical medicine, 2020, 9(5):

30. MU F, WU H, WANG Y. A Retrospective Study: The Significance of Combined Testing of Serum Markers for Diagnosis of Rheumatoid Arthritis [J]. Medical science monitor basic research, 2017, 23(295-303.

31. GRUSZEWSKA E, CYLWIK B, GIŃDZIEŃSKA-SIEŚKIEWICZ E, et al. Diagnostic Power of Galectin-3 in Rheumatic Diseases [J]. Journal of clinical medicine, 2020, 9(10):

32. DUBUCQUOI S, SOLAU-GERVAIS E, LEFRANC D, et al. Evaluation of anti-citrullinated filaggrin antibodies as hallmarks for the diagnosis of rheumatic diseases [J]. Annals of the rheumatic diseases, 2004, 63(4): 415-9.

33. GREINER A, PLISCHKE H, KELLNER H, et al. Association of anti-cyclic citrullinated peptide antibodies, anti-citrullin antibodies, and IgM and IgA rheumatoid factors with serological parameters of disease activity in rheumatoid arthritis [J]. Annals of the New York Academy of Sciences, 2005, 1050(295-303.

34. NI M, ZHOU X, LV Q, et al. Radiomics models for diagnosing microvascular invasion in hepatocellular carcinoma: which model is the best model? [J]. Cancer imaging : the official publication of the International Cancer Imaging Society, 2019, 19(1): 60.

35. WASSERMAN A M. Diagnosis and management of rheumatoid arthritis [J]. American family physician, 2011, 84(11): 1245-52.

\section{Tables}

Table 1

Basic characteristics of the study population 


\begin{tabular}{|c|c|c|c|c|c|c|}
\hline & \multirow{2}{*}{$\begin{array}{l}\text { RA group } \\
(n=158)\end{array}$} & \multirow{2}{*}{$\begin{array}{l}\text { Disease } \\
\text { controls } \\
\bigotimes n=113 \rrbracket\end{array}$} & \multirow{2}{*}{$\begin{array}{l}\text { Healthy } \\
\text { controls } \\
\bigotimes n=45 \rrbracket\end{array}$} & \multicolumn{3}{|c|}{$P$ values for the groups } \\
\hline & & & & $\begin{array}{l}\text { RA vs disease } \\
\text { controls }\end{array}$ & $\begin{array}{l}\text { RA vs healthy } \\
\text { controls }\end{array}$ & $\begin{array}{l}\text { RA vs all } \\
\text { controls }\end{array}$ \\
\hline Gender, n (\%) & $128 \rrbracket 81.01 \rrbracket$ & $100 \rrbracket 88.50 \rrbracket$ & $31 \rrbracket 68.89 \rrbracket$ & 0.096 & 0.082 & 1.000 \\
\hline Age (years) & $52.63 \pm 12.69$ & $51.21 \pm 14.41$ & $55.22 \pm 7.48$ & 0.392 & 0.194 & 0.754 \\
\hline $\mathrm{SAA} 4(\mu \mathrm{g} / \mathrm{ml})$ & $\begin{array}{l}\text { 62.53囚53.05, } \\
76.37 \rrbracket\end{array}$ & $\begin{array}{l}44.03 \rrbracket 31.08, \\
53.26 \rrbracket\end{array}$ & $\begin{array}{l}39.15 \rrbracket 28.39 \\
48.77 \rrbracket\end{array}$ & $<0.001$ & $<0.001$ & $<0.001$ \\
\hline CRP (mg/L) & $\begin{array}{l}12.75 \rrbracket 3.66 \\
39.13 \rrbracket\end{array}$ & $\begin{array}{l}4.60 \rrbracket 3.00 \\
9.10 \rrbracket\end{array}$ & $\begin{array}{l}3.50 \rrbracket 2.15 \\
4.20 \rrbracket\end{array}$ & $<0.001$ & $<0.001$ & $<0.001$ \\
\hline $\begin{array}{l}\text { anti-CCP, pos } \\
(\%)\end{array}$ & $114 \llbracket 72.15 \rrbracket$ & $21 \otimes 18.58 \rrbracket$ & $0 \otimes 0 \otimes$ & $<0.001$ & $<0.001$ & $<0.001$ \\
\hline $\mathrm{RF}(\mathrm{IU} / \mathrm{mL})$ & $\begin{array}{l}51.90(17.23 \\
169.25)\end{array}$ & $\begin{array}{l}8.0 \rrbracket 3.90 \\
21.95 \rrbracket\end{array}$ & $\begin{array}{l}\text { 8.40冈7.10, } \\
9.40 \rrbracket\end{array}$ & $<0.001$ & $<0.001$ & $<0.001$ \\
\hline \multicolumn{7}{|l|}{$\begin{array}{l}\text { Comorbidity, } \\
\text { n (\%) }\end{array}$} \\
\hline Diabetes & $15 \bigotimes 9.50 \rrbracket$ & $2 \otimes 1.76 \rrbracket$ & O४०४ & 0.010 & 0.032 & 0.001 \\
\hline Hypertension & $24 \rrbracket 15.19 \rrbracket$ & $10 \rrbracket 8.95 \rrbracket$ & $0 \otimes 0 \bigotimes$ & 0.120 & 0.005 & 0.011 \\
\hline Smoke, n (\%) & & & & 0.063 & 1.000 & 0.081 \\
\hline Past/Current & $12 \rrbracket 7.59 \rrbracket$ & $2 \otimes 1.76 \rrbracket$ & $3 \rrbracket 6.67 \rrbracket$ & & & \\
\hline Never & 146ه92.41区 & $111 \otimes 98.23 \rrbracket$ & $42 \varangle 93.33 \rrbracket$ & & & \\
\hline Drink, n (\%) & & & & 0.147 & 1.000 & 0.252 \\
\hline Past/Current & $5 \rrbracket 3.16 \rrbracket$ & $0 \otimes 0 \bigotimes$ & $2 \rrbracket 4.44 \rrbracket$ & & & \\
\hline Never & 153凶96.84囚 & 113ه100ه & 43凶95.56ه & & & \\
\hline
\end{tabular}

Table 2

Clinical evaluation of SAA4, CRP, anti-CCP and RF 


\begin{tabular}{|c|c|c|c|c|c|c|c|}
\hline & Sn & $\mathrm{Sp}$ & Youden Index & LR+ & LR- & PPV & NPV \\
\hline \multicolumn{8}{|c|}{ RA group vs disease controls } \\
\hline SAA4 & $67.72 \%$ & $84.96 \%$ & 0.53 & 4.50 & 0.38 & 0.86 & 0.65 \\
\hline CRP & $58.22 \%$ & $71.68 \%$ & 0.30 & 2.06 & 0.58 & 0.74 & 0.55 \\
\hline Anti-CCP & $72.15 \%$ & $81.42 \%$ & 0.54 & 3.88 & 0.34 & 0.84 & 0.67 \\
\hline RF & $71.52 \%$ & $74.33 \%$ & 0.46 & 2.79 & 0.38 & 0.79 & 0.65 \\
\hline \multicolumn{8}{|c|}{ RA group vs healthy controls } \\
\hline SAA4 & $67.72 \%$ & $97.78 \%$ & 0.66 & 30.50 & 0.33 & 0.99 & 0.46 \\
\hline CRP & $58.22 \%$ & $95.56 \%$ & 0.54 & 13.11 & 0.44 & 0.98 & 0.39 \\
\hline Anti-CCP & $72.15 \%$ & $100 \%$ & 0.72 & - & 0.28 & 1.00 & 0.51 \\
\hline RF & $71.52 \%$ & $97.78 \%$ & 0.69 & 32.22 & 0.29 & 0.99 & 0.49 \\
\hline \multicolumn{8}{|c|}{ RA group vs all controls } \\
\hline SAA4 & $67.72 \%$ & $88.60 \%$ & 0.56 & 5.94 & 0.36 & 0.86 & 0.73 \\
\hline CRP & $58.22 \%$ & $78.48 \%$ & 0.37 & 2.71 & 0.53 & 0.73 & 0.65 \\
\hline Anti-CCP & $72.15 \%$ & $86.71 \%$ & 0.59 & 5.43 & 0.32 & 0.84 & 0.76 \\
\hline RF & $71.52 \%$ & $81.01 \%$ & 0.53 & 3.77 & 0.35 & 0.79 & 0.74 \\
\hline
\end{tabular}

Table 3

Clinical evaluation results of SAA4, CRP, anti-CCP and RF 


\begin{tabular}{|llll|}
\hline & Sensitivity & Specificity & Yorden Index \\
\hline SAA4+CRP & $40.51 \%$ & $97.47 \%$ & 0.380 \\
\hline SAA4+anti-CCP & $48.73 \%$ & $98.10 \%$ & 0.468 \\
\hline SAA4+RF & $51.26 \%$ & $95.56 \%$ & 0.468 \\
\hline CRP+ anti-CCP & $43.67 \%$ & $94.94 \%$ & 0.386 \\
\hline CRP+RF & $42.41 \%$ & $94.30 \%$ & 0.367 \\
\hline CCP+RF & $63.92 \%$ & $95.57 \%$ & 0.595 \\
\hline SAA4/CRP & $85.44 \%$ & $69.62 \%$ & 0.551 \\
\hline SAA4/anti-CCP & $91.14 \%$ & $77.22 \%$ & 0.684 \\
\hline SAA4/RF & $90.51 \%$ & $74.51 \%$ & 0.650 \\
\hline CRP/anti-CCP & $86.71 \%$ & $70.25 \%$ & 0.570 \\
\hline CRP/RF & $87.34 \%$ & $65.19 \%$ & 0.525 \\
\hline Anti-CCP/RF & $79.75 \%$ & $72.15 \%$ & 0.519 \\
\hline SAA4+CRP+anti-CCP & $31.65 \%$ & $99.37 \%$ & 0.310 \\
\hline SAA4+CRP+RF & $30.38 \%$ & $98.73 \%$ & 0.291 \\
\hline SAA4+anti-CCP+RF & $43.04 \%$ & $99.37 \%$ & 0.424 \\
\hline CRP+ anti-CCP+RF & $39.87 \%$ & $99.37 \%$ & 0.392 \\
\hline SAA4/CRP/anti-CCP & $96.84 \%$ & $63.29 \%$ & 0.601 \\
\hline SAA4/CRP/RF & $96.20 \%$ & $59.49 \%$ & 0.557 \\
\hline SAA4/anti-CCP/RF & $93.04 \%$ & $66.46 \%$ & 0.595 \\
\hline CRP/anti-CCP/RF & $91.77 \%$ & $60.76 \%$ & 0.525 \\
\hline SAA4+CRP+anti-CCP+RF & $29.11 \%$ & $99.37 \%$ & 0.285 \\
\hline SAA4/CRP/ anti-CCP/RF & $97.47 \%$ & $56.33 \%$ & 0.538 \\
\hline
\end{tabular}

Table 4

Baseline characteristics in training cohort and validation cohort 


\begin{tabular}{|c|c|c|c|c|c|c|}
\hline & \multicolumn{2}{|c|}{ Training cohort } & \multicolumn{4}{|c|}{ Validation cohort } \\
\hline & $\begin{array}{l}\text { RA } \\
\text { group }(n=112)\end{array}$ & $\begin{array}{l}\text { Control } \\
\text { group }(n=112)\end{array}$ & $P$ & RA group $(n=46)$ & $\begin{array}{l}\text { Control } \\
\text { group }(n=46)\end{array}$ & $P$ \\
\hline Gender & & & 1.00 & & & 1.000 \\
\hline male & 22 & 22 & & 8 & 8 & \\
\hline female & 90 & 90 & & 38 & 38 & \\
\hline Age & $53.14 \pm 12.80$ & $52.88 \pm 13.19$ & 0.908 & $51.39 \pm 12.47$ & $51.07 \pm 12.32$ & 0.879 \\
\hline SAA4 $(\mu \mathrm{g} / \mathrm{ml})$ & $66.01(54.34,78.18)$ & $42.87(30.24,52$ & 0.000 & $58.66(51.51,68.91)$ & $42.10(29.41,49.28)$ & 0.000 \\
\hline SAA4+ & $80(71.43 \%)$ & (0) & & $27(58.70 \%)$ & $43(93.48 \%)$ & \\
\hline SAA4- & $32(28.57 \%)$ & $97(86.61 \%)$ & & $19(41.30 \%)$ & $3(6.52 \%)$ & \\
\hline CRP(mg/L) & $11.90(3.51,42.30)$ & $4.30(3.00,7.85)$ & 0.000 & $13.80(4.08,36.75)$ & $3.45(2.60,6.85)$ & 0.000 \\
\hline CRP+ & $64(57.14 \%)$ & $25(22.32 \%)$ & & $28(60.87 \%)$ & $9(19.57 \%)$ & \\
\hline CRP- & $48(42.86 \%)$ & $87(77.68 \%)$ & & 18(39.13\%) & $37(80.43 \%)$ & \\
\hline Anti-CCP(n\%) & & & 0.000 & & & 0.000 \\
\hline Anti-CCP+ & $85(75.89 \%)$ & 16(14.29\%) & & $29(63.04 \%)$ & $5(10.87 \%)$ & \\
\hline Anti-CCP- & $27(24.11 \%)$ & $96(85.71 \%)$ & & 17(36.96\%) & 41(89.13\%) & \\
\hline $\mathrm{RF}(\mathrm{n} \%)$ & & & 0.000 & & & 0.000 \\
\hline RF+ & $82(73.21 \%)$ & $23(20.54 \%)$ & & $31(67.39 \%)$ & 7(15.22\%) & \\
\hline RF- & $30(26.79 \%)$ & $89(79.46 \%)$ & & 15(32.61\%) & $39(84.78 \%)$ & \\
\hline Diabetes(n\%) & & & 0.003 & & & 0.557 \\
\hline Diabetes & 13(11.61\%) & $2(1.79 \%)$ & & $2(4.35 \%)$ & $1(2.17 \%)$ & \\
\hline Non- Diabetes & $99(88.39 \%)$ & 110(98.21\%) & & $44(95.65 \%)$ & $45(97.83 \%)$ & \\
\hline Hypertension(n\%) & & & 0.000 & & & 0.049 \\
\hline Hypertension & 18(16.07\%) & $9(8.04 \%)$ & & $6(13.04 \%)$ & $1(2.17 \%)$ & \\
\hline $\begin{array}{l}\text { Non- } \\
\text { Hypertension }\end{array}$ & $94(83.93 \%)$ & 103(91.96\%) & & $40(86.96 \%)$ & $45(97.83 \%)$ & \\
\hline Smoke(n\%) & & & 0.181 & & & 0.557 \\
\hline Yes & 10(8.93\%) & $5(4.46 \%)$ & & $2(4.35 \%)$ & $1(2.17 \%)$ & \\
\hline No & 102(91.07\%) & 107(95.54\%) & & $44(95.65 \%)$ & $45(97.83 \%)$ & \\
\hline Drink(n\%) & & & 0.175 & & & 1.000 \\
\hline Yes & $4(3.57 \%)$ & $1(0.89 \%)$ & & $1(2.17 \%)$ & $1(2.17 \%)$ & \\
\hline No & 108(96.43\%) & 111(99.11\%) & & 45(97.83\%) & 45(97.83\%) & \\
\hline
\end{tabular}

Table 5

Univariate logistic regression and multivariate logistic regression models in the training cohort $(n=224 \rrbracket$ 


\begin{tabular}{|c|c|c|c|c|}
\hline & \multicolumn{2}{|c|}{ Univariate logistic regression } & \multicolumn{2}{|c|}{ Multivariate logistic regression } \\
\hline & OR $(95 \% \mathrm{CI})$ & $P$ & OR $(95 \% \mathrm{Cl})$ & $P$ \\
\hline SAA4 & 16.17(8.18 31.94) & 0.000 & $14.25(5.73 \sim 35.44)$ & 0.000 \\
\hline CRP & $4.64(2.60 \sim 8.30)$ & 0.000 & $2.92(1.24 \sim 6.91)$ & 0.014 \\
\hline Anti-CCP & 18.89 (9.53 37.43) & 0.000 & 10.61(4.20 26.81ه & 0.000 \\
\hline RF & 10.58(5.69 19.67) & 0.000 & 4.02(1.65 9.82) & 0.002 \\
\hline Diabetes & 7.22(1.59 32.80) & 0.010 & $14.39(1.81 \sim 114.46)$ & 0.012 \\
\hline Hypertension & $2.19(0.94 \sim 5.12)$ & 0.070 & & \\
\hline Smoke & $2.10(0.69 \sim 6.35)$ & 0.019 & & \\
\hline Drink & $4.11(0.45 \sim 37.37)$ & 0.209 & & \\
\hline
\end{tabular}

\section{Figures}
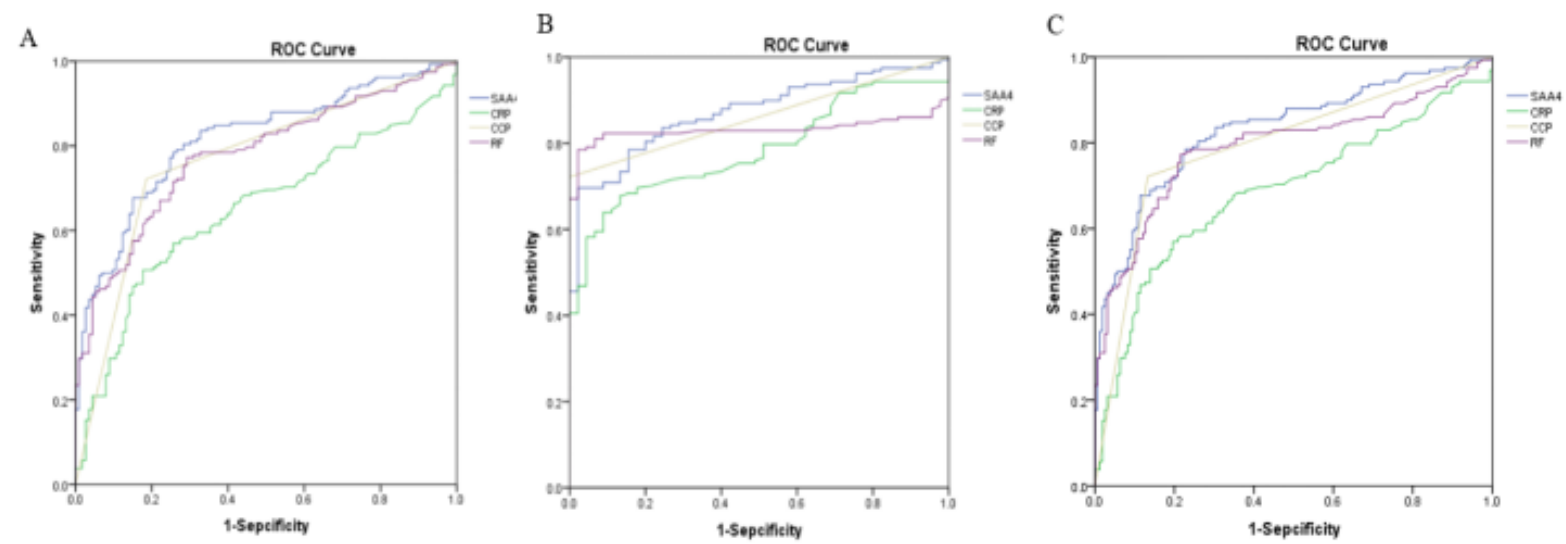

Figure 1

\section{ROC of SAA4, CRP, anti-CCP and RF for RA diagnosis in comparison of different groups.}

A. ROC of SAA4, CRP, anti-CCP and RF when comparing RA groups and disease controls. AUC of SAA4 is 0.83 [95\% $\mathrm{Cl}$ : 0.79 0.88]; AUC of CRP is 0.69[95\% Cl:0.63-0.75]; AUC of anti-CCP is 0.77[95\% Cl: 0.71-0.83]; AUC of RF is 0.79 [95\% $\mathrm{Cl}$ : 0.74-0.85];

B. ROC of SAA4, CRP, anti-CCP and RF when comparing RA groups and healthy controls.

AUC of SAA4 is 0.87[95\% Cl: 0.82-0.92]; AUC of CRP is 0.78[95\% Cl: 0.72-0.85]; AUC of anti-CCP is 0.86[95\% Cl: 0.81-0.91]; AUC of $\mathrm{RF}$ is $0.83[95 \% \mathrm{Cl}$. 0.78-0.89];

C. ROC of SAA4, CRP, anti-CCP and RF when comparing RA groups and all controls. AUC of SAA4 is $0.83[95 \% \mathrm{Cl}$ : 0.77-0.88]; AUC of CRP is 0.65 [95\% Cl: $0.58-0.71$ ]; AUC of anti-CCP is 0.77 [95\% Cl: $0.71-0.83$ ]; AUC of RF is 0.78 [95\% $\mathrm{Cl}$ : $0.72-0.83$ ] 

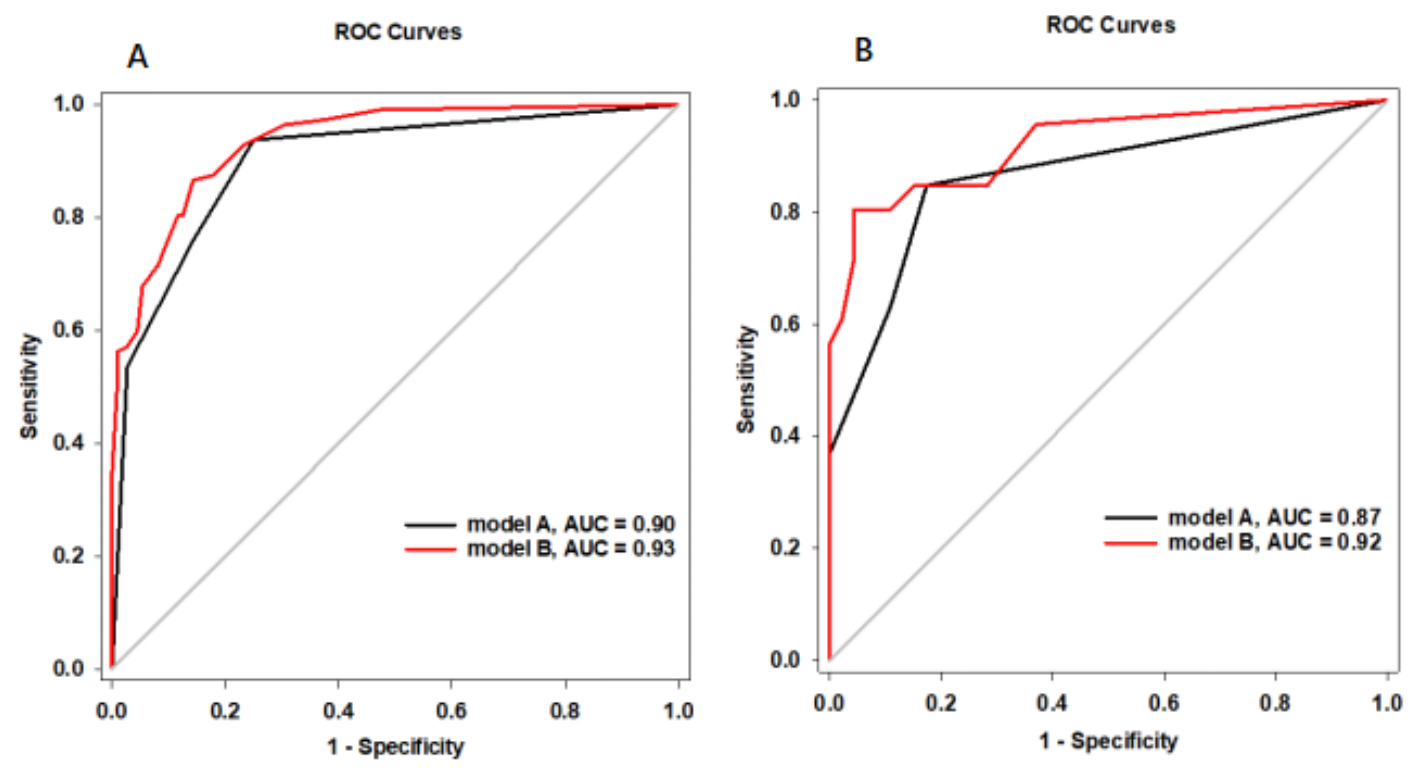

Figure 2

ROC curves for the diagnostic models.

A. AUC of mode I A is $0.90[95 \% \mathrm{Cl}: 0.86-0.94]$ in training cohort. AUC of model $\mathrm{B}$ is $0.93[95 \% \mathrm{Cl}: 0.91-0.96]$ in training cohort.

B. AUC of model A is $0.87[95 \% \mathrm{Cl}$ : $0.78-0.94]$ in validation cohort. AUC of model $\mathrm{B}$ is $0.92[95 \% \mathrm{Cl}: 0.87-0.97]$ in validation cohort.

A

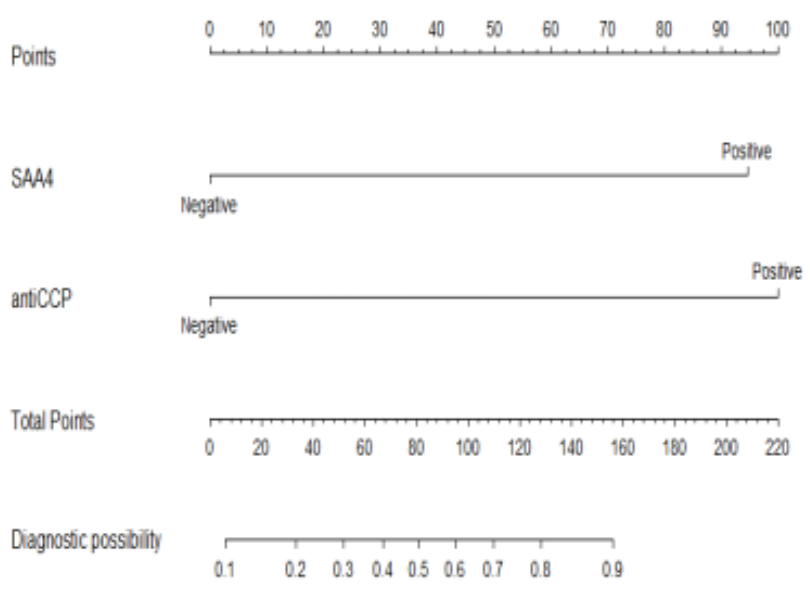

B

Points

SAA4

CRP

$\operatorname{artcCP}$

RF

Total Points

Diagnostic possibitity
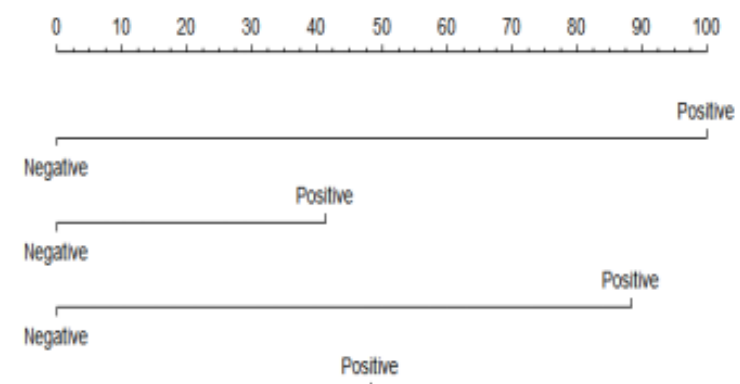

Negative

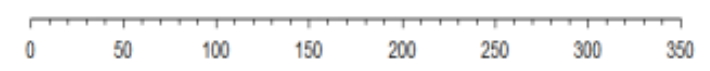

\begin{tabular}{llllllll}
\hline 0.1 & 0.2 & 0.30 .4 & 0.5 & 0.6 & 0.7 & 0.8 & 0.9
\end{tabular}

Figure 3

Nomogram to predict the probability of RA.

A. developed nomogram of model A in the training cohort.

B. developed nomogram of model B in the training cohort. 
A

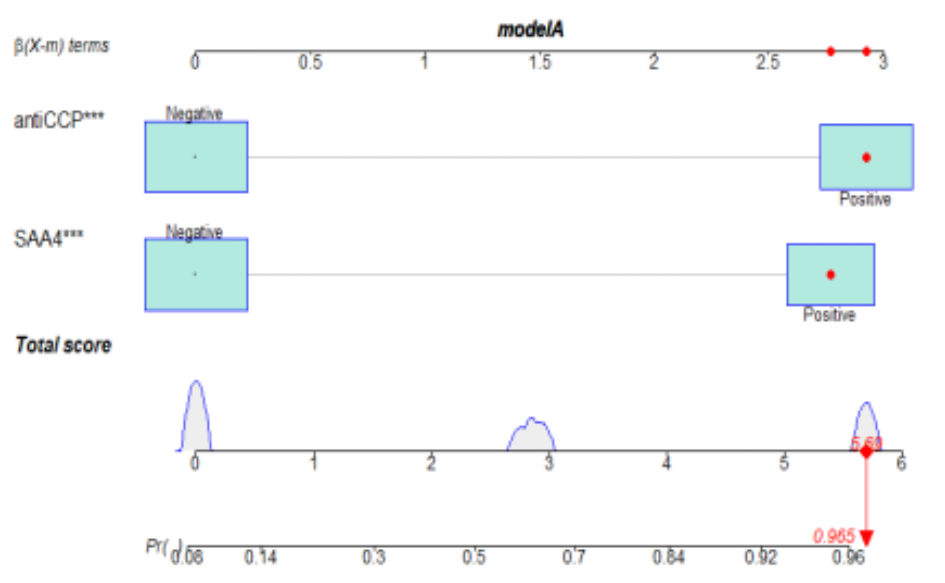

B

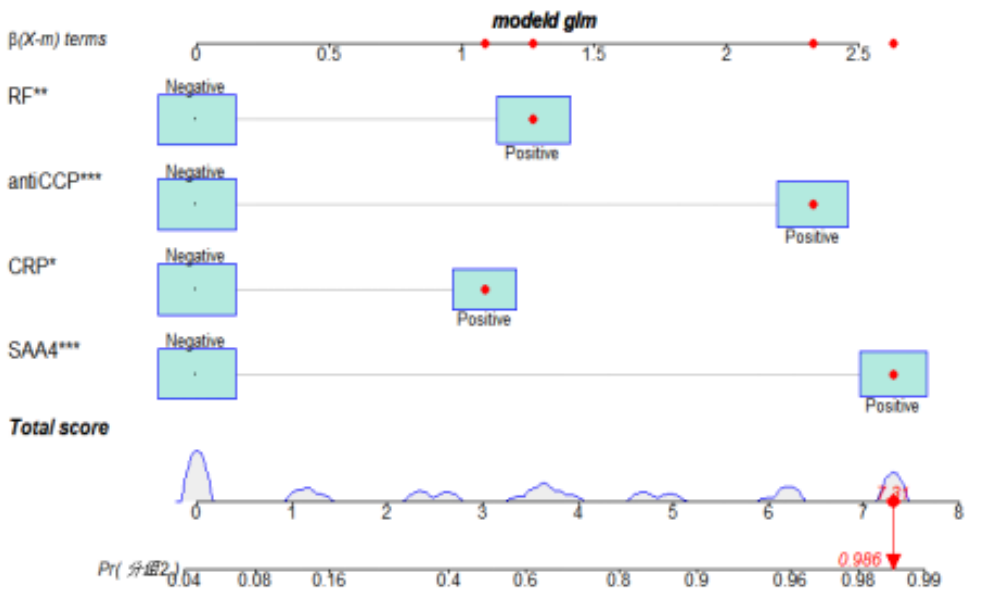

\section{Figure 4}

Nomogram to predict the probability of RA.

A. Example prediction nomogram of model A for diagnosing RA

B. Example prediction nomogram of model B for diagnosing RA 